\title{
Erinnerung an die Zukunft
}

Der Pazifische Ozean als Herausforderung für die europäische

\author{
Moderne - am Beispiel ,transpazifischer Texte von
}

Egon Erwin Kisch, Vicki Baum und Alfred Döblin (1933-1946)

\section{JOHANNES GÖRBERT}

\begin{abstract}
The following article investigates assessments of the Pacific Ocean as the ssea of the future,, following a Mediterranean past and an Atlantic present, and standing in a stark contrast to the rexotic<, marginalised view of its islands and shores. It analyses three German-language texts from the era of the Second World War: Egon Erwin Kisch's travelogue Secret China (China geheim, 1933), Vicki Baum's Hotel Shanghai (1939), and Alfred Döblin's short expository text The Golden Gate (Das Goldene Tor, 1946). Despite the many variations in their portrayals of wars and crises, migrations and acculturations, breakdowns and new beginnings around the Pacific Ocean, all three authors evaluate the challenge posed by the transpacific triangle evolving around China, Japan, and the US to Europe's self-postulated role as a leading continent. In that vein, they are (re-)activating a specific lieu de mémoire of a Pacific future in world affairs, in hopes of heightening transcultural self-understanding and self-positioning in times of crisis.
\end{abstract}

Title: Remembering the Future. The Pacific Ocean as a Challenge to European Modernity - the Example of >Transpacific « Texts by Egon Erwin Kisch, Vicki Baum and Alfred Döblin (1933-1946)

Keywords: Baum, Vicki (1888-1960); Döblin, Alfred (1878-1957); Kisch, Egon Erwin (1885-1948); lieux de mémoire; Pacific Ocean

\section{Der Pazifik als transkultureller Erinnerungsort}

Der Pazifische Ozean ist im (trans-)kulturellen Gedächtnis der Gegenwart in zwei sehr unterschiedlichen, ja geradezu gegensätzlichen Konzeptionen präsent. Zum einen gelten die zahllosen, weitverstreuten Pacific Islands im tropischeren Teil des Ozeans spätestens seit der Südseemode des 18. Jahrhunderts als vermeintliche Idylle, als ein letztes Refugium, wo ein Leben abseits einer durchrationalisierten modernen Welt noch möglich erscheint. Zum anderen - und diese zweite Konzeption steht im Mittelpunkt dieses Beitrags - gilt das größte Weltmeer gerade nicht als ein Gegenpol zur Moderne, sobald sich der Blick auf den vom vulkanischen Ring of Fire umschlossenen Pacific Rim und auf den Nordpazifik richtet, dorthin, wo sich die ostasiatischen Länder China und Japan am West- und die Westküste der USA am Ostufer des Großen Ozeans gegenüberlie- 
gen. Dass gerade aktuell im frühen 21. Jahrhundert ein am sogenannten Stillen Ozean sich zuspitzender Atomkonflikt Schlagzeilen macht, dass erst kürzlich in der Außenpolitik der noch einzigen verbliebenen Supermacht der Erde von »America's Pacific Century« die Rede war (vgl. Görbert/Schwarz 2017: 15) und, ganz entscheidend, dass China, Japan und die USA in all ihren Verflechtungen aktuell die weltweit drei größten Volkswirtschaften mit einer atemberaubenden ökonomischen Dynamik stellen: All dies veranlasst Betrachter des Weltgeschehens immer wieder dazu, den pazifischen Raum als einen Kristallisationspunkt, als ein Machtgravitationszentrum wahrzunehmen, in dessen Richtung sich die entscheidenden Schwergewichte in Weltpolitik, Weltwirtschaft und Weltgesellschaft mehr und mehr zu verschieben anschicken. Nach einer mediterran geprägten Welt der Vormoderne und einer lange Zeit (trans-)atlantisch akzentuierten Moderne sehen die Betrachter des Zeitgeschehens nunmehr eine »pazifische Epoche « heraufziehen, deren Aufstieg sie mit Diagnosen von europäischen und auch deutschen Szenarien des Abstiegs verknüpfen (vgl. als nur ein Beispiel Seifert 2015). Der pazifische Raum avanciert daher nicht nur geographisch, sondern auch kultursymbolisch bzw. in Urteilen zu transkulturellen Wechselbeziehungen zu einem Antipoden, zu einer entscheidenden Herausforderung für die europäische Moderne.

Das Anliegen der nachfolgenden Textanalysen ist es, aufzuzeigen, dass diese gegenwärtig diskutierte Konzeption des Pazifiks als zukünftiges >Mittelmeer der Moderne < keineswegs neu ist, sondern dass es sich letztendlich um einen aktualisierten Erinnerungsort im kulturellen Gedächtnis Europas und Deutschlands handelt, der weit bis ins 19. Jahrhundert zurückreicht. So taucht diese Prognose etwa bereits in Wilhelm Raabes Roman Die Leute aus dem Walde von 1863 auf. Dort heißt es ganz ähnlich über die nahende weltpolitische Zukunft: »In jener Zeit werden gewaltige neue Nationen auf riesenhaften Schiffen zwischen den Ufern Asiens und Amerikas verkehren, wie jetzt zwischen Hull und Hamburg, Dover und Calais «, und weiter: »Da wird die Zivilisation ihren Lauf um den Erdball vollendet haben, und das alte Europa, einst eine so schöne, blühende Jungfrau, [...] wird dann ein vertrocknetes Mütterlein sein, das [...] alte Schätze und Andenken in altväterlichen Kommoden [...] und in der Schürze hält« (Raabe 1971: 364). Erst durch den Einbezug dieser historischen Tiefendimension erhellt sich somit, dass die Debatte über den Pazifik als schicksalsträchtigen maritimen $\mathrm{Zu}$ kunftsraum einen der zahlreichen kulturdiagnostischen Knotenpunkte im gesellschaftlichen Diskurs in Europa darstellt, der bevorzugt dann (re-)aktiviert wird, wenn es um die Selbstverständigung und -positionierung in Krisenzeiten geht. Im Anschluss an Pierre Noras Ansatz der lieux de mémoire interessiert somit maßgeblich das »symbolic element«, das diesem Diskurs inhärent ist und das in letzter Konsequenz als Teil des »memorial heritage« (Nora 1996: XVII) von kulturellen Gemeinschaften verstanden werden kann. Ziel meines Beitrags ist es in diesem Sinne, anhand dieses maritimen pazifischen Diskurses »nicht mehr die [historischen] Ereignisse an sich« genauer zu untersuchen, »sondern deren Konstruktion in der Zeit, das Verschwinden und Wiederaufleben ihrer Bedeutungen« bzw. »ihre ständige Wiederverwendung« sowie ihren »Bedeu- 
tungsgehalt « und die Art, wie Diskurstraditionen zu verschiedenen Zeiten »geschaffen und weitergegeben « werden (Nora 2005: 16; vgl. auch Siebeck 2017). Ein besonderes Augenmerk liegt hierbei auf transkulturellen Konstellationen: einerseits im Verhältnis zwischen pazifischen Ziel- und europäischen Herkunftsräumen; zum anderen aber auch in den Beziehungsgeflechten im stranspazifischen Dreieck < zwischen China, Japan und den USA, welche die Texte besonders anhand von urbanen Settings in den Weltmetropolen San Francisco, Shanghai und Tokyo genauer beleuchten.

Historisch fokussiert sich der Beitrag auf die erste Hälfte des 20. Jahrhunderts und damit auf einen Zeitraum, in dem der Pazifik weltpolitisch ganz besonders an Brisanz gewinnt. Dementsprechend intensiv wird das größte Weltmeer seinerzeit diskutiert, auch und speziell aus deutschsprachiger Perspektive. Die hier herangezogenen Textbeispiele werden dabei in den 1930er und 1940er Jahren unmittelbar vor, während und nach dem Zweiten Weltkrieg geschrieben. Es handelt sich um den Reisebericht China geheim von Egon Erwin Kisch von 1933, um den Roman Hotel Shanghai von Vicki Baum von 1939 und um Alfred Döblins kurzes Geleitwort zu der von ihm herausgegebenen Literaturzeitschrift Das Goldene Tor von 1946. Die daraus behandelten Ausschnitte sind Bestandteile einer Anthologie, die im Forschungsprojekt »Transpacifica « an der Freien Universität Berlin und an der University of Tokyo (unter meiner Mitbeteiligung) erarbeitet wurde und deren Veröffentlichung bevorsteht. Diese Anthologie setzt an bei der Jahrhundertwende um 1900 und enthält zusätzlich zu den literarischen Texten Artikel aus der Tagespresse und Publizistik sowie Beiträge aus der Wissenschaft und den darstellenden Künsten (vgl. den Artikel zum Projekt von Huggins 2017). Im Mittelpunkt steht die deutschsprachige Auseinandersetzung mit den transkulturellen Beziehungen am und zum Großen Ozean im Zeitalter des Imperialismus und der beiden Weltkriege: in einer Zeit, in der immer zahlreichere Stimmen laut werden, die eine entscheidende Verlagerung der machtpolitischen Mitte des Erdballs in das >transpazifische Dreieck < prophezeien.

\section{Krieg und Krise: Egon Erwin Kisch, China geheim}

Als Egon Erwin Kisch im Frühling/Sommer 1932 für mehrmonatige Recherchen zu einem Band seiner >rasenden Reportagen< (vgl. einführend Bůžek 2016) illegal mit der Transsibirischen Eisenbahn nach China einreist, sieht er sich dort gleich mehrfach mit Krieg und Krise konfrontiert (vgl. zu den Reiseumständen Patka 1999: 125f., sowie allgemein zu Kischs schriftstellerischem »Mobilitätsprogramm« Uecker 2001: 155). Erstens begibt er sich in ein Land, das sich schon seit fünf Jahren in einem Bürgerkrieg befindet, der erst 1949 mit dem Sieg der Kommunisten unter Mao enden sollte. Zweitens wird Kisch nachträglich zum Zeitzeugen des sogenannten Shanghai-Zwischenfalls, einer militärischen Auseinandersetzung zwischen Japan und China im Kontext der Mandschurei-Krise, deren Kämpfe von Ende Januar bis Anfang Mai 1932 andauerten. Kisch behandelt diese Gemengelage von vornherein als multilateralen Konflikt von weltpo- 
litischer Tragweite. Für ihn symbolisiert Shanghai wie kaum ein zweites seiner Reiseziele die zunehmende Ohnmacht der >alten « gegenüber dem Aufschwung der >neuen < Weltmächte. Bei aller Kontinuität von imperialistischer Ausbeutung und Unterdrückung, die China in der ersten Hälfte des 20. Jahrhunderts zu erleiden hat, zeichnet sich dennoch, so sein Reisebericht, ein elementarer Wandel in der »permanenten Krise« und »Unmoral des Kapitalismus« (Patka 1998: 284) ab. Am Beispiel Shanghais lässt es sich hierbei besonders markant aufzeigen, wie sich die Akzente der Weltpolitik epochal in Richtung Pazifik verlagern. Dementsprechend sieht sich Kisch in einem Interview im Anschluss an seine Rückkehr nach Europa wie »in eine andere Welt versetzt; man ist aus der Zukunft in die Vergangenheit, aus dem Leben in eine sterbende Welt gekommen!« (Kisch zit. n. Patka 1999: 126)

Alles in allem legt Kisch, gerade mit Bezug auf die »politischen Spannungen« und »eskalierenden Konflikte« in der pazifischen Küstenstadt, seinem Reisebericht ein »Konzept der Gegensätzlichkeit« Shanghais zugrunde (Xu 2015: 193). Während einerseits die japanische Armee die chinesischen Stadtviertel in Schutt und Asche legt, sehen sich andererseits die europäischen Ausländer in den international settlements von Shanghai im Gegenzug auf die Rolle so paralysierter wie gedankenloser Zuschauer zurückgeworfen. Sie betrachten den Krieg, so Kisch, »wie aus einer Proszeniumsloge«:

[M]an [merkte] im Settlement fast nichts davon, daß nebenan Greuel auf Greuel sich begab. Schiffe, Straßenbahnen, Rikschas fuhren ihre Bahn, Kinos spielten, Firmen handelten, Zollbehörden amtierten, Zeitungen erschienen, dieweil am Firmament Granaten einander kreuzten, dieweil Straßenzüge brannten, dieweil Kinder von zusammenkrachenden Häusern begraben wurden, dieweil Familien flüchteten und dieweil immer wieder, immer wieder Menschen getroffen zu Boden sanken. (Kisch 1977: 407f.)

Deutlicher könnte der Kontrast kaum ausfallen: Hier die gleichförmige Alltagsbetriebsamkeit der Europäer, dort die weltpolitische Dramatik, mit der China und Japan einen blutigen Straßen- und Häuserkampf mitten unter und zulasten der Zivilbevölkerung Shanghais austragen.

Ebenso hilf- und teilnahmslos bzw. grotesk statusbewusst wie die Bewohner der settlements lässt auch die eigens für die Kämpfe in China eingesetzte LyttonKommission des Völkerbunds, benannt nach ihrem Vorsitzenden, dem ehemaligen Gouverneur und Vizekönig von Indien, Victor Bulwer-Lytton (1876-1947), die Militärs in Shanghai gewähren. Das Interesse ihres Besuchs liegt laut Kischs Darstellung ohnehin weniger auf einer kritischen Untersuchung der Vorfälle als auf exquisiten diplomatischen »Empfängen, Tees, Diners und Soupers « (ebd.: 408), bei denen die Delegation sich ausgerechnet von Japanern als »den Veranstaltern des Krieges « fürstlich bewirten lässt. »Es gab sechzehnerlei Weine und Sekte, Upman-Zigarren (Ladenpreis 1 Dollar 6o, in eingeschliffenen Rundgläsern aus Havanna importiert) und eine ausreichende Speisenfolge« (ebd.). Dieser Luxus allein reicht schon aus, um die ausschließlich >westlich < zusammengesetzte Delegation ebenso großzügig wie heuchlerisch über die Verheerungen 
in Shanghai hinwegsehen $\mathrm{zu}$ lassen. Kischs China-Reportage versteht sich somit »in gewisser Weise als Gegenbericht« zu dem »kontroverse[n] Bericht«, den die Kommission zuvor über die Mandschurei-Krise im Allgemeinen und die Kämpfe um Shanghai im Speziellen vorgelegt hatte: »Sie hat einen investigativen Charakter. Mit fremder Identität getarnt besucht der Reporter den Ort des Geschehens, Shanghai, um sich ein eigenes Bild von der Situation zu machen« (Streim 2011: 168). Kischs Fazit fällt eindeutig aus: Während er, wie typisch für seine Reportagen, für die Außenseiter und Unterdrückten, hier für die von Krieg und Gewalt Geplagten, Partei ergreift, überzieht er die Völkerbundkommission mit beißendem »Hohn und Spott« (Gruner 1992: 183) angesichts ihrer demonstrativen Untätigkeit und Dekadenz an einem Brennpunkt des Weltgeschehens.

Auch die Rolle Deutschlands in der Welt sieht Kisch in Shanghai symptomatisch geschwächt. Zum Sinnbild dafür erhebt er die Zerstörung der vom Wilhelminischen Kaiserreich gegründeten Tongji-Universität in den japanisch-chinesischen Kämpfen. Kisch schreibt hierzu:

Auch die deutsche Universität von Tungchi ist zerschossen. [...] Japan zielte hierher, Japan nahm auf die schwarzrotgoldene Flagge keine Rücksicht [...]. Eine Fliegerbombe wurde in die Maschinenhalle geworfen, aus Schiffsgeschützen ins Physiologische Institut gepfeffert, ins Auditorium Maximum, in die Klinik und in die Dozentengebäude. In der Mitte des Fußballplatzes sind jetzt zum Scherz und dennoch mit deutscher Gründlichkeit alle Granathülsen aufgestellt wie Kegel. (Kisch 1977: 409)

Ein deutsches Prestigeprojekt am Pazifik gerät an dieser Stelle gleich auf mehreren Ebenen >unter Beschuss «: ganz wortwörtlich durch das Kreuzfeuer der japanischen Luft-, See- und Landstreitkräfte sowie im übertragenen Sinne dadurch, dass die neue, siegreiche Inselweltmacht aus dem >Fernen Osten< eine der >eigenen < und gar als >besonders zivilisiert < eingeschätzten Tugenden karikaturesk übersteigert. Einen ähnlichen Prozess der Machtverlagerung deutet Kisch an anderer Stelle schon mit seiner Wortwahl zum Kriegsgeschehen an: »Gefangene wurden nicht gemacht. Pardon wurde nicht gegeben « (ebd.: 407). Hier zitiert der Reporter, nur leicht abgewandelt, aus der berühmt-berüchtigten >Hunnenrede`, mit welcher der deutsche Kaiser Wilhelm II. seinerzeit zur rücksichtslosen Niederschlagung des chinesischen Boxeraufstands um 1900 aufrief (vgl. Xu 2015: 200). Indem Kisch diese Worte circa dreißig Jahre später nicht mehr deutschen, sondern japanischen Militärführern in den Mund legt, verweist er zum einen auf eine bezeichnende Beständigkeit imperialen Machtmissbrauchs. Zum anderen betont er aber auch hier, dass die weltpolitische Initiative eben nicht mehr bei den europäischen, sondern bei den pazifischen Machthabern liegt.

Anders als die Briten und die Deutschen, und im Übrigen auch die Franzosen, Italiener und weiteren Europäer, aus denen sich die internationalen Stadtbezirke und ebenfalls die Mitglieder der Lytton-Kommission zusammensetzen, sind es zumindest die Kennzeichen US-amerikanischer Macht, die die Japaner in Shanghai nachhaltig zu beeindrucken vermögen. So registriert Kisch aufmerksam den »Verkehr der Kontraste« im Hafen: »Da begegnen einander 
die größten Dreadnoughts der Welt und die kleinsten Fischerboote der Welt. 10.000 Tonnen faßt das amerikanische Flaggschiff >Houston<, jedes seiner Geschoße ist größer als jeder der Sampans, die es umschwärmen« (Kisch 1977: 406). Neben diesem stahlgewordenen Inbegriff von Militärmacht sind es die »silbernen Tanks von Shell, von Standard Oil, von Texas Oil« (ebd.) am Ufer des >Bunds < von Shanghai, die auch die wirtschaftliche Macht der USA am Pazifik eindrucksvoll verkörpern. Auf diese Gebäude wagen die japanischen Streitkräfte ganz bewusst keinen Angriff - und das, obwohl ihre Militäroffensive, wie sie Kisch beschreibt, weite Teile Shanghais wie ein »frisch erzeugte[s] Pompeji« (ebd.: 407) aussehen lässt. Wo zuvor die europäischen Kolonialmächte federführend unterdrückten, ausbeuteten und zerstörten, knüpfen jetzt Pazifikanrainer wie Japan und die USA direkt an ihre Vorläufer an - mit ebenso latenter wie manifester Präsenz ihrer Militär- und Wirtschaftskräfte (vgl. zur direkten Konkurrenz zwischen Japan und den USA am Stillen Ozean auch Sommadossi 2021).

Für diese >neuen « wie für die >alten « Anwälte der Fremdherrschaft« (Kisch 1977: 514) hat Kisch in China geheim nur Verachtung übrig. Nach seinem Urteil haben sie die Bevölkerung Chinas

[nur] am Leben gelassen, um aus ihr Konzessionen und Kontributionen, Indemnitäten und Realitäten herauszupressen, aus ihr einträgliche Objekte für Opium und Morphium, Korruption und Prostitution zu formen, aus inr Zugtiere und Haustiere und Arbeitstiere zu machen [...] und ihren Boden zu besetzen. In den inneren Kämpfen, den Bürgerkriegen, haben die Fremden stets die Partei der einheimischen Unterdrücker genommen, nachdem sie beiden Seiten für gutes Geld Waffen geliefert hatten. (Ebd.)

Kischs Hoffnungen richten sich, wie bei anderen >linken< Autoren seiner Zeit auch, auf ein >erwachendes < China unter kommunistischer Führung, das sich in Zukunft vom Imperialismus emanzipieren und sich erneut als »Weltreich « zu etablieren vermag: Mit Shanghai als »Welthafen« und »Mündungsgebiet des $>$ Kaisers der Ströme«: dem Jangtsekiang als dem »1000 Meilen lang schiffbaren einzigen Weg zu einem Zehntel der Menschheit« (ebd.). Dass diese Voreingenommenheit für den Kommunismus in Spannung steht zum entschiedenen Anspruch Kischs auf journalistische Unparteilichkeit, ja dass sie an vielen Stellen gar »Fakten-Fiktionen im Namen der gewissen Wahrheit« (Schütz 2006: 79; vgl. ähnlich Schütz 2008: 200) hervorgebracht haben mag, ändert nichts Grundsätzliches an der Schärfe seiner Gegenwartsanalyse. In Kischs Reisebericht tritt nicht (mehr) ein exotisches, alteritäres oder ein gestriges bzw. schlichtweg nicht ernstzunehmendes China in den Vordergrund (vgl. ähnlich Felbert 1986: 141), sondern eine Arena der Realpolitik, in deren Kämpfen entscheidende Weichen für die Zukunft der Welt gestellt werden. 


\section{Migration und Akkulturation: Vicki Baum, Hotel Shanghal}

Auch bei Vicki Baum fungiert die chinesische Weltstadt >über dem Meer < als ganz wortwörtlicher Fluchtpunkt ihrer literarischen Darstellung einer >Neuen Mitte <am Pazifik. Baums Text, der zunächst als Fortsetzungsroman in der Zeitschrift Cosmopolitan erschien und im Zusammenhang mit einer regelrechten »Shanghai-Mode in der europäisch-amerikanischen Belletristik « in der ersten Hälfte des 20. Jahrhunderts steht, läuft als »Faction« (Aurnhammer 2006: 215, 221) auf das historische Ereignis des Zweiten Chinesisch-Japanischen Krieges (1937-1945) zu. Zu dessen anfänglichen >Kollateralschäden< zählte der Bombenanschlag vom 14. August 1937 auf das internationale Shanghai-Hotel am >Bund<, dem insgesamt neun Menschen zum Opfer fielen. »In den folgenden Seiten soll Bericht gegeben werden über die Wege, die diese neun Menschen nach Shanghai brachten, über den Verlauf ihres Lebens und über die Stunde ihres Todes« (Baum 2007: 8), so kündigt die Autorin in der Einleitung ihre Zielsetzung einer sogenannten group novel an (siehe einführend zu diesem Genre Scherer 2012: 126-128). Dabei entscheidet sie sich für eine Zweiteilung ihres Romans. »Die Menschen«, der erste Teil, rekapituliert die nach Shanghai führenden transkulturellen Lebensläufe der neun Protagonisten, während »Die Stadt«, der zweite Teil, mit der für Baum narratologisch »typische[n] Erzählsituation [...] im Kontinuum zwischen Nullfokalisierung [...] und variablen internen Fokalisierungen « (Nottelmann 2002: 296) ein räumliches Panorama der Weltmetropole ausbreitet. Bemerkenswert an Baums Roman ist, dass Shanghai darin als doppelt international anschlussfähig dargestellt wird: zum einen als eine Stadt, $z u$ der hin sich die ganze Welt versammelt, zum anderen als ein Verkehrsknotenpunkt, von dem aus sich ein weltumspannendes Beziehungsgeflecht entspinnt (vgl. dazu auch Aurnhammer 2006: 215; Scherer 2018: 141). Ähnlich oder sogar mehr noch als in Kischs Reisebericht liegt es im Interesse von Baums Roman, abstrakte Prozesse von globaler Relevanz in den Einzelschicksalen ihrer Protagonisten anschaulich werden zu lassen.

Für transpazifische Erkenntnisinteressen besonders einschlägig ist der Werdegang von Yoshio Murata, dem einzigen Japaner unter Baums neun Protagonisten. Bei Murata handelt es sich um einen feinfühligen, hochgebildeten Menschen, der, nach einer Erziehung im Zeichen altehrwürdiger Samuraiwerte, sein Heimatland für eine Berufsausbildung zum Journalisten Richtung USA verlässt und von dort aus schlussendlich als Kriegsreporter zurück über den Pazifik nach Shanghai beordert wird. »Er war unsicher und rastlos, wie alle Menschen, die in zwei verschiedenen Kulturen leben« (Baum 2007: 258), so schildert Baum die Disposition ihres in seiner Perspektive auf sich selbst als »heimatlos« empfindenden (Stephan 2011: 191; vgl. ähnlich Xu 2015: 363), mehrfach über das Meer verschlagenen Protagonisten. Einmal in den USA angekommen, sieht sich Murata zahlreichen Kulturschocks ausgesetzt, die aber nicht etwa an einer möglicherweise mangelhaften Vorbildung oder an einer grundsätzlichen Unvertrautheit mit einem modernen Lebensalltag liegen. Im Gegenteil: 
Vierzehn Jahre lang hatte er Englisch studiert, an der Wand seines Zimmers zu Hause klebte ein Gedicht von Walt Whitman [...]. Er war [...] ein Kenner der Literatur, der selbst Gedichte [...] zu schreiben verstand, ein verlegener Philosoph mit Brille und Fotografenapparat. Er wußte die statistischen Zahlen des Warenaustauschs zwischen Japan und Amerika auswendig, und er konnte [Herbert] Spencer [den Evolutionstheoretiker, der die Formel des Survival of the Fittest prägte; J.G.] seitenweise zitieren. (Baum 2007: 253f.).

Gleichermaßen wie mit Sprache, Politik, Wirtschaft und Kultur zeigt sich Murata mit dem Alltag seines Gastlands vertraut, sogar stärker, als es ihm im Grunde genommen lieb ist.

Obwohl er nicht zu viel erwartet hatte, wurde er ein leises Enttäuschtsein nicht los. Häuser, Maschinen, Zeitungen und Straßen waren eigentlich nicht anders als zu Hause. Der junge Japaner war heimisch in Kinopalästen, Drugstores und Sodafountains, in Klubs und Bibliotheken [...]. So gut hatte Japan den Westen nachgeahmt, daß es keine Überraschungen mehr übrig ließ. (Ebd.: 255)

Im Ergebnis steht ein Lebenslauf zwischen einer fernöstlichen Insel- und einer fernwestlichen Kontinentalkultur, der gerade nicht auf unüberbrückbare Differenzen hinausläuft. Vielmehr kreiert Baums Roman mit Murata ein Individuum zwischen zwei Nationen, das sich im Bildungsgut beider Seiten bewandert zeigt (und nebenbei auch ein Faible für europäische Literatur nachweist, vgl. etwa ebd.: 245 u. 251) und das sich stilsicher auf dem Boden zweier pazifischer Nationen bewegt, die analog zueinander den Kurs einer entschiedenen Modernisierung eingeschlagen haben.

Selbst die Momente kultureller Irritation, die Murata in Amerika durchlebt, unterscheiden sich kaum von denen westlicher Exilanten in den USA. Zwar leidet er als Japaner zweifelsohne stärker als andere Ausländer an der asiatenfeindlichen Einwanderungspolitik seines Gastlands und an Momenten rassistischer Diskriminierung im Alltag. Und doch stößt sich Murata vor allem an dem, was auch westliche Beobachter an Amerika immer wieder kritisieren: zum Beispiel an der rastlosen Geschäftigkeit, Oberflächlichkeit, Anonymität, Grobheit und dem Militarismus der US-amerikanischen Gesellschaft. Doch selbst in den Augenblicken, in denen Murata Japan im Gegenzug als »das feinste, schönste, edelste und liebenswerteste Land der Welt« (Baum 2007: 258) verklärt, relativiert Baums Roman sein Heimweh direkt im Anschluss als ein nur allzu menschliches, universales Gefühl: »Aber so ging es jedem Menschen, den er kannte, mit seiner eigenen Heimat [...]. Der nationale Hochmut, mit dem er ausgezogen war, schliff sich ab, je mehr er von der Welt sah.« (Ebd.) Murata wird damit zum Bestandteil einer Weise der Figurencharakterisierung, auf die Baum auch bei ihren weiteren Protagonisten in Hotel Shanghai Sorgfalt verwendet und die »eine Reihe von Beispielen für lebendige Akkulturationsprozesse « sowie eine »prinzipielle Offenheit dem Anderen gegenüber « und »neue hybride Existenzformen« (Sommersacher 2013: 230; vgl. ähnlich Aurnhammer 2006: 224) vorführt. Im 
Ergebnis steht in Hotel Shanghai das Porträt eines transkulturellen, hier ostasiatischen Werdegangs, der sich in seiner Aufgeklärtheit und Modernität weit von jeglichen Exotismen von Japan als dem ganz Anderen der westlichen Kultur entfernt - und der seine Lebens- und Karriereziele eben nicht mehr mit einem prioritären Blick nach Europa (das Murata nur einmal im Schnelldurchlauf bereist, vgl. Baum 2007: 259-263), sondern in den Ländern des transpazifischen Dreiecks zu realisieren anstrebt.

\section{Zusammenbruch und NeuAnfang: Alfred Döblin, Das Goldene Tor}

Derartige Szenarien für Krieg und Krise, für Migration und Akkulturation sind auch Alfred Döblin als dem dritten hier vorgestellten >transpazifischen alles andere als fremd. Schließlich erlebte er den Zweiten Weltkrieg selbst als Exilant an der kalifornischen Westküste der Vereinigten Staaten; und schließlich arbeitete Döblin >west-östliche< Kampfplätze in gleich mehreren seiner Romane detailliert heraus. Bereits in seinem Debüt Die drei Sprünge des Wang-lun (1916) beschäftigte sich Döblin bekanntlich mit dem Thema der inneren Unruhen im >Reich der Mitte<; und auch in Berge, Meere, Giganten (1924), in dem ein Pazifikkrieg im 26. Jahrhundert geschildert wird, und in seinem letzten Roman Hamlet oder Die lange Nacht nimmt ein Ende (1956), in dem ein japanischer Kamikazeangriff die Romanhandlung eröffnet, zeigt sich Döblin stark interessiert an Schlachten um die Weltherrschaft, die er im und am Pazifik ansiedelt.

Ganz verschieden davon funktioniert jedoch der kurze Text über Das Goldene Tor, der hier als letztes Beispiel für den Pazifik als Herausforderung für die europäische Moderne in den Fokus gerückt werden soll. Mit diesem »Geleitwort« eröffnet Döblin 1946 die gleichnamige, von ihm begründete Monatsschrift für Literatur und Kunst, die er, die meiste Zeit mit Unterstützung der Behörden in der französischen Besatzungszone Südwestdeutschlands, bis 1951 herausgab (vgl. zum Programm und zur Geschichte der Zeitschrift Birkert 1989). In der kleinen Schrift, mit ihrem als »pathetisch-hoffnungsfro[h]« (Sander 2001: 77) oder gar als »etwas übertrieben optimistisch« (Mombert 2006: 203) erscheinenden Duktus, imaginiert Döblin den Pazifik eben nicht als Kriegsschauplatz, sondern als Ausgangspunkt eines künftigen Weltfriedens. Als dessen Grundlage begreift er die Gründung der Vereinten Nationen mit der Unterzeichnung ihrer Charta durch 51 Nationen am 26. Juni 1945 in San Francisco, die er selbst noch als in Kalifornien Ansässiger miterlebt hatte. Dass dieser Gründungsakt gerade nicht am Atlantik, sondern am Pazifik stattfand, bewertet Döblin als ausdrucksstarkes neues »Symbol für die menschliche Freiheit und die Solidarität der Völker« (Döblin 2005: 226) im Rahmen einer neuen Weltordnung. In diesem Zusammenhang lässt er andere Assoziationen in Richtung der >Alten Welt < zurücktreten, welche die Titelwahl und die Emblematik des Zeitschriftentitelbildes ebenfalls grundsätzlich zulassen: Hat die Forschung doch darauf aufmerksam gemacht, dass ein »Goldene[s] Tor« (hebräisch >Tor des Erbarmens $<$ ) auch »in der Altstadt Je- 
rusalems« (Hoff 2016: 250) zu finden ist und dass zudem die Illustration des Tores auf dem Titelblatt, »durch das Sonnenstrahlen wie Schienen dringen; im Hintergrund helle Wolken, eine gerade Linie als Andeutung der Meeresbucht, dazu ein Lebensbaum« (Schoeller 2011: 659), auch als »antikisieren[d]« bzw. als »Strahlen der Aufklärung in einer modernen Variante « (Hoff 2016: 250) gedeutet werden könnten. Auch die »Zeilen der Lyrikerin Emma Lazarus, wie sie an der Sockelinschrift der amerikanischen Freiheitsstatue [ergo: an einem der Symbolstätten der >Neuen Welt <; J.G.] zu finden sind « (ebd.), harmonieren durchaus mit dem von Döblin gewählten Titel: »Send these, the homeless, tempest-tossed to me / I lift me lamp beside the golden door « (zit. n. ebd.).

Und doch setzt Döblin in seinem Geleitwort alles daran, einen weltpolitischen Neuanfang fast gänzlich ohne Rekurse auf das Erbe bzw. die Modernisierung Europas im Zeichen von Antike, jüdisch-christlichen Wurzeln und Aufklärung sowie ohne eine Priorisierung des transatlantischen Bündnisses (auf das sich schließlich im Allgemeinen die westeuropäische wie im Speziellen die bundesdeutsche Nachkriegspolitik einschworen) in Szene zu setzen. Sein Goldenes Tor verweist, wie Döblin auch in seinem Journal 1952/53 ausführt, zuallererst nicht nach Jerusalem oder New York, sondern auf »die herrliche Einfahrt, the Golden Gate« von San Francisco, auf »die Verbindung der Neuen Welt mit Asien« (Döblin 1986: 397). Der Autor wendet sich damit gegen die gerade unmittelbar nach dem Zweiten Weltkrieg wieder sehr einflussreiche >Abendlandidee<, deren Konzept von »Weltoffenheit« für gewöhnlich »unter der Bedingung einer resakralisierten und als geschlossene Ordnung zu denkenden Welt« (KepplerTasaki 2018: 17) diskutiert wurde. Anstelle einer solchen Leitidee für die Nachkriegsordnung sieht Döblin »das hellere Licht« für die Zukunft »aus der transpazifischen Vermittlung zwischen asiatischer Weisheit und amerikanischer Jugendlichkeit« bzw. aus dem »lebendigen Kontakt mit der indo- und asienpazifischen Region« kommen (ebd.: 18f.; vgl. für Döblins Distanz zur >Abendlandidee auch Mombert 2006: 214). Für ihn muss nicht nur »das alte Europa«, sondern auch die »Ostflanke des [amerikanischen] Kontinents« (Döblin 2005: 225) mit seiner größten Stadt New York seine Rolle als führender Ort der sogenannten >freien Welt< an den Pazifik abtreten. So wird in Döblins New York Freiheit weniger progressiv neu ausbuchstabiert als vielmehr konservativ »bewach $[\mathrm{t}] \ll$ : mit Wolkenkratzern, die sich »wie eine Riesenschildwache [...] aufgestellt haben« und die, mit ihren »steinernen, grauen Kronen«, eine »undurchdringliche Mauer « bilden, mit einer »erschütternden Wucht beladen« sind sowie am Abend »auf der Lauer [liegen] und [...] aus zehntausend hellen flimmernden Augen auf das Meer hinaus[schauen]« (ebd.). An diesem Befestigungsort wird die Freiheit eben schon länger nicht mehr visionär ausgestaltet, sondern wehrhaft gegen die »Tyrannei« (ebd.) verteidigt: zum Beispiel in der Nachfolge des Marquis de La Fayette (1757-1834), der sich sowohl im Amerikanischen Unabhängigkeitskrieg als auch in den Kämpfen der Französischen Revolution als General profilierte.

Ein ganz anderes Porträt zeichnet Döblin von der Stadt des Heiligen Franziskus, den er als den »Freund aller Menschen« und als den »Bruder der Armen, des Feuers, der Sonne, des Wassers und sogar des Todes« (ebd.: 226) vorstellt - 
ein dezenter Fingerzeig auf die christliche Prägung des 1941 in Kalifornien zum Katholizismus konvertierten Autors und damit auch seiner Zeitschrift (vgl. Hoff 2016: 253). Im Hafen von San Francisco, »an dem dunstumwobenen Goldenen Tor, läßt man ein« (Döblin 2005: 226). Hier erwartet den Besucher kein Bollwerk, sondern eine »Brücke, fein wie Filigran«, umgeben von einem »zarte[n], schmerzlich weiche[n] Hauch, der unsere Seelen rührt und [der] an Ferne, Weite und Zukunft denken läßt« (ebd.). Insofern ist es nur folgerichtig, dass es exakt die Milde dieses Ortes ist, »welche die Menschen zwingt, eben noch Krieger, sich hinzusetzen und sich ernsthaft das Versprechen zu geben, zusammenzuhalten und über den Frieden zu wachen, nun dennoch wieder. Sie sind nicht über Nacht Engel geworden, aber sie können nicht umhin zu zeigen, daß sie mehr als ein Stück Natur sind.« (Ebd.)

In dieser pazifischen, nicht mehr in der atlantischen Szenerie sieht Döblin nach »diesem beispiellosen, die ganze Erde umlaufenden Wald- und Präriebrand « zuerst »sich wieder im Grunde das Leben [regen], das gute Leben in den Wurzeln der verbrannten Pflanzen« (ebd.). »Die ganze Realität ist anders« (ebd.), mit dieser Perspektive auf eine >Neue Mitte< möchte Döblins Goldenes Tor Zyniker zum Schweigen bringen, die in der Geschichte immer nur die Wendung zum Schlechteren bzw. die Wiederkehr der immer gleichen Fehler am immer gleichen Ort erkennen. Am Pazifik, so Döblin, sehen sich »die großen und kleinen Nationen, gedenkend ihrer Toten«, vereint, »um die Freiheit zu schützen und die Völker zusammenzuschließen« (ebd.). An dieser »Einfahrt zur Neuen Welt vom alten Asien her« (ebd.) erscheint nun ein Projekt möglich, an dem Europa bis 1945 immer wieder gescheitert ist: eine friedliche, eben pazifistische Weltpolitik, ausgehend von der fernab des Atlantiks neugegründeten internationalen peacekeeping organization der United Nations. Gegenüber dem »kulturellen Kompass der Abendlandidee « sind die »Himmelsrichtungen [...] in dieser Lagebeschreibung « Döblins signifikant »verschoben: Europa kommt in einem verdächtigen >Osten $<$ zu stehen, der >Westen $<$ im Sinne von Menschlichkeit und Freiheit, Zukunft und Produktivkraft ist an den Pazifik gewandert.« (KepplerTasaki 2018: 18)

\section{Zukunfts- und Erinnerungsraum Pazifik}

Es zeigt sich somit, dass sich Diskussionen über die künftige Rolle Deutschlands und Europas in der Welt nicht allein am Thema der Meere entzünden, die den >Alten Kontinent< selbst umströmen. Vielmehr ist es hier, in der kriegsgeschüttelten ersten Hälfte des 20 . Jahrhunderts, gerade der alteritäre, antipodische Pazifik, der die Autoren dazu veranlasst, das >Mittelmeer der Moderne $<$ nicht mehr in der mediterranen oder transatlantischen Nähe, sondern mehr und mehr in der Ferne, im transpazifischen Dreieck zwischen China, Japan und den USA zu lokalisieren. Bei Egon Erwin Kisch verdichtet sich diese Herausforderung der >alten< durch eine >neue< Mitte am Kriegs- und Konfliktschauplatz des >schlafenden < bzw. >erwachenden Riesens < China. In dessen Weltmetropole Shanghai 
lassen die Streit- und Wirtschaftskräfte Japans und der USA ihre Muskeln spielen, während die europäischen Mächte nur noch wie paralysiert zusehen können. Bei Vicki Baum rückt neben der Kriegs- auch die Migrationsproblematik ins Zentrum. Ihr Protagonist Yoshio Murata verkörpert den hohen Bildungsstand und die interkulturelle Adaptionsfähigkeit eines modernen Intellektuellen, für dessen Werdegang der Blick nach Europa nur noch eine sehr marginale Rolle spielt. Und bei Alfred Döblin schließlich richten sich die Hoffnungen auf eine zukünftige Nachkriegsweltordnung, die für mehr Frieden, Stabilität und Prosperität sorgen können soll als die vergangene Politik der Mächte diesseits und jenseits des Atlantiks. Für ihn ist es nur konsequent, dass sich diese Weltordnung weit entfernt von New York, London und Paris eben am >Goldenen Tor<, im pazifischen San Francisco, konstituiert: an einem eindrucksvollen, neuartigen Symbolort für die Verständigung der Nationen. Allen hier vorgestellten Texten ist dabei gemeinsam, dass sie - wie zahllose weitere Kulturzeugnisse vor und nach ihnen spätestens seit dem 19. Jahrhundert und bis in unsere Gegenwart hinein - den Pazifik stets aufs Neue als einen lieu de mémoire heranziehen, sobald ganz grundsätzlich transkulturelle Standortbestimmungen in Zeiten globaler Krisen gefragt sind.

\section{LITERATUR}

Aurnhammer, Achim (2006): Vicki Baums Hotel Shanghai (1939) im Kontext der deutschen Shanghai-Romane. In: Maoping Wei/ Wilhelm Kühlmann (Hg.): Deutsch-chinesische Literaturbeziehungen. Shanghai, S. 214-235.

Baum, Vicki (2007): Hotel Shanghai. Roman [1939]. Köln.

Birkert, Alexandra (1989): Das Goldene Tor. Alfred Döblins Nachkriegszeitschrift (Rahmenbedingungen, Zielsetzung, Entwicklung). In: Archiv für Geschichte des Buchwesens 33, S. 201-317.

Bůžek, Karolin (2016): Einführung: Die literarische Reportage des Egon Erwin Kisch oder 'Die Reportage der Sensationen،. In: Viera Glosíková/Sina Meißgeier/Ilse Nagelschmidt (Hg.): Im Einzelschicksal die Weltgeschichte: Egon Erwin Kisch und seine literarischen Reportagen. Berlin, S. 11-15.

Döblin, Alfred (1986): Ausgewählte Werke in Einzelbänden [1952/1953]. Bd. 17: Schriften zu Leben und Werk. Hg. v. Erich Kleinschmidt. Olten/Freiburg.

Ders. (2005): Das Goldene Tor [1946]. In: Ders.: Ausgewählte Werke in Einzelbänden. Bd. 20: Kleine Schriften IV. Hg. v. Anthony W. Riley u. Christina Althen. Olten/Freiburg, S. $225 f$.

Felbert, Ulrich von (1986): China und Japan als Impuls und Exempel. Fernöstliche Ideen und Motive bei Alfred Döblin, Bertolt Brecht und Egon Erwin Kisch. Frankfurt a.M. u.a.

Görbert, Johannes/Schwarz, Thomas (2017): Einführung. In: Dies./Mario Kumekawa (Hg.): Pazifikismus. Poetiken des Stillen Ozeans. Würzburg, S. 13-42. 
Gruner, Fritz (1992): Egon Erwin Kisch und China. Eine Betrachtung zum Buch China geheim ein halbes Jahrhundert nach seinem Erscheinen. In: Adrian Hsia (Hg.): Fernöstliche Brückenschläge. Zu deutsch-chinesischen Literaturbeziehungen im 20. Jahrhundert. Bern u.a., S. 179-188.

Hoff, Dagmar von (2016): [Art.] "Die Zeitschrift Das Goldene Tor (1946-1951)“. In: Sabina Becker (Hg.): Döblin Handbuch. Leben - Werk - Wirkung. Stuttgart, S. 250-254.

Huggins, Jonas (2017): Das große neue Mittelmeer. Furcht und Hoffnung: Wie die Deutschen von 1900 bis 1945 auf den Pazifik blickten. In: Der Tagesspiegel v. 2. Dezember 2017, S. B8.

Keppler-Tasaki, Stefan (2018): Berliner Heimat. Alfred Döblins proletarischer Kosmopolitismus. In: Text + Kritik. Zeitschrift für Literatur, H. 13/14 (Neufassung): Alfred Döblin. Hg. v. Sabine Kyora, S. 3-21.

Kisch, Egon Erwin ( ${ }^{21977): ~ Z a r e n, ~ P o p e n, ~ B o l s c h e w i k e n . ~ A s i e n ~ g r u ̈ n d l i c h ~ v e r a ̈ n d e r t . ~ C h i-~}$ na geheim [1933]. Gesammelte Werke in Einzelausgaben. Bd. 3. Hg. v. Bodo Uhse u. Gisela Kisch. Berlin/Weimar.

Mombert, Monique (2006): "Das Inokulieren frischer Keime zur Anregung eines neuen Wachstums" in der Zeitschrift Das Goldene Tor. In: Dies./Christine Maillard (Hg.): Der Grenzgänger Alfred Döblin, 1940-1957. Biographie und Werk. Internationales Alfred-Döblin-Kolloquium Strasbourg 2003. Bern u.a., S. 199-215.

Nora, Pierre (1996): From Lieux de mémoire to Realms of Memory. Preface to the English-Language Edition. In: Ders./Lawrence D. Kritzman (Hg.): Realms of Memory. Rethinking the French Past. Bd. 1. New York, S. XV-XXIV.

Ders. (2005): Wie läßt sich heute eine Geschichte Frankreichs schreiben? [1993]. In: Ders. (Hg.): Erinnerungsorte Frankreichs. Aus dem Franz. v. Michael Beyer. München, S. 15-23.

Nottelmann, Nicole (2002): Strategien des Erfolgs. Narratologische Analysen exemplarischer Romane Vicki Baums. Würzburg.

Patka, Marcus G. (1998): Facetten rasender Zeit. Der Schriftsteller Egon Erwin Kisch hinter der Maske des Reporters. In: Ders. (Hg.): Egon Erwin Kisch. Eine Biographie in Bildern. Berlin, S. 273-287.

Ders. (1999): Egon Erwin Kisch. Stationen im Leben eines streitbaren Autors. Wien.

Raabe, Wilhelm (1971): Sämtliche Werke. Braunschweiger Ausgabe. Bd. 5: Die Leute aus dem Walde [1863]. Hg. v. Karl Hoppe. Göttingen.

Sander, Gabriele (2001): Alfred Döblin. Stuttgart.

Scherer, Stefan (2012): Globalisierung in der Zwischenkriegszeit. China im Weltstadtroman des Exils: Vicki Baums Hotel Shanghai (1939). In: Uwe Japp/Aihong Jiang (Hg.): China in der deutschen Literatur 1827-1985. Berlin u.a., S. 125-141.

Ders. (2018): Weltstadtroman Hotel Shanghai (1939) von Vicki Baum. In: Tamara Kudryavtseva u.a. (Hg.): Germanistik zwischen Tradition und Innovation. Akten des XIII. Kongresses der Internationalen Vereinigung der Germanistik (IVG) Shanghai 2015. Bd. 9: Tradition und Transformation: Der Ferne Osten in der deutschsprachigen Literatur. Frankfurt a.M. u.a., S. 139-142.

Schoeller, Wilfried F. (2011): Alfred Döblin. Eine Biographie. München. 
Schütz, Erhard (2006): Der (g)rasende Reporter oder Merkwürdige Wahrheiten des Egon Erwin Kisch. In: Klaus Siebenhaar (Hg.): Die Sprache der Bilder. Hermann Haarmann zum 60. Geburtstag. Berlin, S. 69-86.

Ders. (2008): Egon Erwin Kisch - Faktograph oder Fiktio-Fürst? In: Andy Hahnemann/David Oels (Hg.): Sachbuch und populäres Wissen im 20. Jahrhundert. Frankfurt a.M. u.a., S. 183-200.

Seifert, Thomas (2015): Die pazifische Epoche: Wie Europa gegen die neue Weltmacht Asien bestehen kann. München.

Siebeck, Cornelia (2017): Erinnerungsorte, Lieux de Mémoire, Version: 1.0. In: Docupedia-Zeitgeschichte, 2.3.2017; online unter http://docupedia.de/zg/Siebeck_erinnerungsorte_v1_de_2017 [Stand 1.10.2020].

Sommadossi, Tomas (2021): Einführung Sektion 6 (Literatur und Essay). In: Ders./Stefan Keppler-Tasaki/ Johannes Görbert (Hg.): Transpacifica. Deutschsprachige Quellen zu den kulturellen und politischen Beziehungen im Pazifikgebiet 1900-1945. München [im Druck].

Sommersacher, Björn (2013): Vicki Baum: Hotel Shanghai (1939). In: Bettina Bannasch/Gerhild Rochus (Hg.): Handbuch der deutschsprachigen Exilliteratur. Von Heinrich Heine bis Herta Müller. Berlin/Boston, S. 226-233.

Stephan, Inge (2011): Bilder und NachBilder vom Exil in Shanghai in Literatur und Film. In: Almut Hille/Gregor Streim/Pan Lu (Hg.): Deutsch-chinesische Annäherungen. Kultureller Austausch und gegenseitige Wahrnehmung in der Zwischenkriegszeit. Wien, S. 187-203.

Streim, Gregor (2011): Das Erwachen des Kulis. China in den Reisereportagen der Weimarer Republik (Richard Huelsenbeck - Arthur Holitscher - Egon Erwin Kisch). In: Ders./Almut Hille/Pan Lu (Hg.): Deutsch-chinesische Annäherungen. Kultureller Austausch und gegenseitige Wahrnehmung in der Zwischenkriegszeit. Wien, S. 155171.

Uecker, Matthias (2001): Leitbild Reporter. Egon Erwin Kischs Medienstrategien. In: Ders./Susanne Marten-Finnis (Hg.): Berlin - Wien - Prag: Moderne, Minderheiten und Migration in der Zwischenkriegszeit/Modernity, Minorities and Migration during the Interwar Period. Bern u.a., S. 143-157.

Xu, Fangfang (2015): "Auch Shanghai hatte sich sehr verändert". Der Wandel des Shanghai-Bildes in der deutschsprachigen Literatur 1898-1949. Würzburg. 